\title{
Article \\ A More Accurate Half-Discrete Hilbert-Type Inequality Involving One upper Limit Function and One Partial Sum
}

\author{
Xianyong Huang ${ }^{1}$, Shanhe $\mathrm{Wu}^{2, *(\mathbb{D})}$ and Bicheng Yang ${ }^{3}$ \\ 1 Department of Mathematics, Guangdong University of Education, Guangzhou 510303, China; \\ huangxianyong@gdei.edu.cn \\ 2 Department of Mathematics, Longyan University, Longyan 364012, China \\ 3 Institute of Applied Mathematics, Longyan University, Longyan 364012, China; bcyang@gdei.edu.cn \\ * Correspondence: shanhewu@lyun.edu.cn
}

check for

updates

Citation: Huang, X.; Wu, S.; Yang, B. A More Accurate Half-Discrete Hilbert-Type Inequality Involving One upper Limit Function and One Partial Sum. Symmetry 2021, 13, 1548. https://doi.org/10.3390/ sym13081548

Academic Editors: Nicusor Minculete and Ioan Rașa

Received: 27 July 2021

Accepted: 18 August 2021

Published: 23 August 2021

Publisher's Note: MDPI stays neutral with regard to jurisdictional claims in published maps and institutional affiliations.

Copyright: (c) 2021 by the authors. Licensee MDPI, Basel, Switzerland. This article is an open access article distributed under the terms and conditions of the Creative Commons Attribution (CC BY) license (https:// creativecommons.org/licenses/by/ $4.0 /)$.

\begin{abstract}
In this paper, by virtue of the symmetry principle, we construct proper weight coefficients and use them to establish a more accurate half-discrete Hilbert-type inequality involving one upper limit function and one partial sum. Then, we prove the new inequality with the help of the EulerMaclaurin summation formula and Abel's partial summation formula. Finally, we illustrate how the obtained results can generate some new half-discrete Hilbert-type inequalities.
\end{abstract}

Keywords: weight coefficient; Euler-Maclaurin summation formula; Abel's partial summation formula; half-discrete Hilbert-type inequality; upper limit function

\section{Introduction}

The celebrated Hardy-Hilbert's inequality reads as:

$$
\sum_{m=1}^{\infty} \sum_{n=1}^{\infty} \frac{a_{m} b_{n}}{m+n}<\frac{\pi}{\sin (\pi / p)}\left(\sum_{m=1}^{\infty} a_{m}^{p}\right)^{\frac{1}{p}}\left(\sum_{n=1}^{\infty} b_{n}^{q}\right)^{\frac{1}{q}},
$$

where $p>1, \frac{1}{p}+\frac{1}{q}=1, a_{m}, b_{n} \geq 0,0<\sum_{m=1}^{\infty} a_{m}^{p}<\infty$ and $0<\sum_{n=1}^{\infty} b_{n}^{q}<\infty$, the constant factor $\frac{\pi}{\sin (\pi / p)}$ is the best possible (see [1], Theorem 315).

A more accurate form of (1) was provided in ([1], Theorem 323), as follows:

$$
\sum_{m=1}^{\infty} \sum_{n=1}^{\infty} \frac{a_{m} b_{n}}{m+n-1}<\frac{\pi}{\sin (\pi / p)}\left(\sum_{m=1}^{\infty} a_{m}^{p}\right)^{\frac{1}{p}}\left(\sum_{n=1}^{\infty} b_{n}^{q}\right)^{\frac{1}{q}} .
$$

In 2006, by introducing parameters $\lambda_{i} \in(0,2](i=1,2), \lambda_{1}+\lambda_{2}=\lambda \in(0,4]$, an extension of (1) was provided by [2] as follows:

$$
\sum_{m=1}^{\infty} \sum_{n=1}^{\infty} \frac{a_{m} b_{n}}{(m+n)^{\lambda}}<B\left(\lambda_{1}, \lambda_{2}\right)\left[\sum_{m=1}^{\infty} m^{p\left(1-\lambda_{1}\right)-1} a_{m}^{p}\right]^{\frac{1}{p}}\left[\sum_{n=1}^{\infty} n^{q\left(1-\lambda_{2}\right)-1} b_{n}^{q}\right]^{\frac{1}{q}},
$$

where the constant factor $B\left(\lambda_{1}, \lambda_{2}\right)$ is the best possible, and the beta function is defined as:

$$
B(u, v)=\int_{0}^{\infty} \frac{t^{u-1}}{(1+t)^{u+v}} d t(u, v>0)
$$

Obviously, when $\lambda=1, \lambda_{1}=\frac{1}{q}, \lambda_{2}=\frac{1}{p}$, inequality (2) reduces to (1); when $p=q=2$, $\lambda_{1}=\lambda_{2}=\frac{\lambda}{2}$, inequality (2) reduces to the inequality presented by Yang in [3].

Recently, applying inequality (3) and Abel's summation by parts formula, Adiyasuren et al. [4] gave a new inequality with the kernel $\frac{1}{(m+n)^{\lambda}}$ involving two partial sums. Inequality (1), 
with its integral analogues, is playing an important role in analysis and its applications (see [5-8]).

In 1934, a half-discrete Hilbert-type inequality was given as follows ([1], Theorem 351): assuming that $K(t)(t>0)$ is a decreasing function, $0<\phi(s):=\int_{0}^{\infty} K(t) t^{s-1} d t<\infty, a_{n} \geq 0$, such that $0<\sum_{n=1}^{\infty} a_{n}^{p}<\infty$, we have:

$$
\int_{0}^{\infty} x^{p-2}\left(\sum_{n=1}^{\infty} K(n x) a_{n}\right)^{p} d x<\phi^{p}\left(\frac{1}{q}\right) \sum_{n=1}^{\infty} a_{n}^{p} .
$$

In 2016, Hong et al. [9] considered some equivalent statements of the extensions of (1) with the best possible constant factor related to several parameters. Some extensions of inequality (4) were given by [10-15]. Recently, Yang et al. [16,17] gave reverse half-discrete Hardy-Hilbert's inequalities and dealt with their equivalent statements of the best possible constant factor related to several parameters.

In this article, following the method of $[2,4,9]$, in the light of the symmetry principle, we construct proper weight coefficients and use them to establish a more accurate half-discrete Hilbert-type inequality involving one upper limit function and one partial sum. Subsequently, we prove this new inequality by means of the Hermite-Hadamard inequality, Euler-Maclaurin summation formula and Abel's partial summation formula. As an extension of the obtained results, the equivalent statements of the best possible constant factor related to several parameters are discussed. It is shown that some new half-discrete Hilbert-type inequalities can be derived from the special cases of our main results.

\section{Some Lemmas}

In what follows, we suppose that $p>1, \frac{1}{p}+\frac{1}{q}=1, \eta \in\left[0, \frac{1}{4}\right], \lambda \in(0,2], \lambda_{1} \in(0, \lambda+1)$, $\lambda_{2} \in\left(0, \frac{1}{2}\right] \cap(0, \lambda+1), \hat{\lambda}_{1}:=\frac{\lambda-\lambda_{2}}{p}+\frac{\lambda_{1}}{q}, \hat{\lambda}_{2}:=\frac{\lambda-\lambda_{1}}{q}+\frac{\lambda_{2}}{p}$. We also assume that $f(x)(\geq 0)$ is a Lebesgue integrable function in any interval $(0, b](b>0)$, and define the upper limit function $F(x):=\int_{0}^{x} f(t) d t(x \geq 0)$ with the partial sums as follows:

$$
A_{n}:=\sum_{k=1}^{n} a_{k}\left(a_{n} \geq 0, n \in \mathrm{N}:=\{1,2, \cdots\}\right),
$$

which satisfies $F(x)=o\left(e^{t x}\right), A_{n}=o\left(e^{t(n-\eta)}\right)(t>0 ; x, n \rightarrow \infty)$ :

$$
0<\int_{0}^{\infty} x^{-p \hat{\lambda}_{1}-1} F^{p}(x)<\infty \text { and } 0<\sum_{n=1}^{\infty}(n-\eta)^{-q \hat{\lambda}_{2}-1} A_{n}^{q}<\infty .
$$

Lemma 1. (i) Let $(-1)^{i} \frac{d^{i}}{d t^{i}} g(t)>0, t \in[m, \infty)(m \in \mathrm{N})$ with $g^{(i)}(\infty)=0(i=0,1,2,3)$, and let $P_{i}(t), B_{i}(i \in \mathrm{N})$ be the Bernoulli functions and the Bernoulli numbers of $i$-order. Then, we have ([5]):

$$
\int_{m}^{\infty} P_{2 q-1}(t) g(t) d t=-\varepsilon_{q} \frac{B_{2 q}}{2 q} g(m)\left(0<\varepsilon_{q}<1 ; q \in \mathrm{N}\right) .
$$

In particular, forq $=1$, in view of $B_{2}=\frac{1}{6}$, we have:

$$
-\frac{1}{12} g(m)<\int_{m}^{\infty} P_{1}(t) g(t) d t<0 ;
$$

For $q=2$, in view of $B_{4}=-\frac{1}{30}$, it follows that:

$$
0<\int_{m}^{\infty} P_{3}(t) g(t) d t<\frac{1}{120} g(m) .
$$


(ii) If $h(t)(>0) \in C^{3}[m, \infty), h^{(i)}(\infty)=0(i=0,1,2,3)$, then we have the following Euler-Maclaurin summation formula:

$$
\sum_{k=m}^{\infty} h(k)=\int_{m}^{\infty} h(t) d t+\frac{1}{2} h(m)+\int_{m}^{\infty} P_{1}(t) h^{\prime}(t) d t
$$

where:

$$
\int_{m}^{\infty} P_{1}(t) h^{\prime}(t) d t=-\frac{1}{12} h^{\prime}(m)+\frac{1}{6} \int_{m}^{\infty} P_{3}(t) h^{\prime \prime \prime}(t) d t .
$$

Lemma 2. Let $s \in(0,4], s_{2} \in\left(0, \frac{3}{2}\right] \cap(0, s), k_{s}\left(s_{i}\right):=B\left(s_{i}, s-s_{i}\right)(i=1,2)$, and let $\omega\left(s_{2}, x\right)$ denote the following weight coefficient:

$$
\mathcal{\omega}\left(s_{2}, x\right):=x^{s-s_{2}} \sum_{n=1}^{\infty} \frac{(n-\eta)^{s_{2}-1}}{(x+n-\eta)^{s}}\left(x \in \mathrm{R}_{+}:=(0, \infty)\right)
$$

Then, we have the following inequalities:

$$
0<k_{s}\left(s_{2}\right)\left(1-O\left(\frac{1}{x^{s_{2}}}\right)\right)<\omega\left(s_{2}, x\right)<k_{s}\left(s_{2}\right),
$$

where we indicate $O\left(\frac{1}{x^{s_{2}}}\right):=\frac{1}{k_{s}\left(s_{2}\right)} \int_{0}^{\frac{1-\eta}{x}} \frac{u^{s_{2}-1}}{(1+u)^{s}} d u>0$.

Proof. For fixed $x \in \mathrm{R}_{+}$, we define a function $g(x, t)$ by:

$$
g(x, t):=\frac{(t-\eta)^{s_{2}-1}}{(x+t-\eta)^{s}}(t \in(\eta, \infty))
$$

which implies that $g(x, t)>0\left(t \in I_{\eta}\right)$ and $g \in C^{\infty}\left(I_{\eta}\right)$, where $I_{\eta}:=(\eta, \infty)$. In the following, we consider two cases of $s_{2} \in(0,1) \cap(0, s)$ and $s_{2} \in\left[1, \frac{3}{2}\right] \cap(0, s)$ to prove inequalities (12).

(i) For $s_{2} \in(0,1) \cap(0, s)$, since:

$$
(-1)^{i} \frac{\partial^{i}}{\partial t^{i}} g(x, t)>0(t>\eta ; i=0,1,2),
$$

by the Hermite-Hadamard inequality, setting $u=\frac{t-\eta}{x}$, we have:

$$
\begin{gathered}
\mathscr{\omega}\left(s_{2}, x\right)=x^{s-s_{2}} \sum_{n=1}^{\infty} g(x, n)<x^{s-s_{2}} \int_{\frac{1}{2}}^{\infty} g(x, t) d t \\
=x^{s-s_{2}} \int_{\frac{1}{2}}^{\infty} \frac{(t-\eta)^{s_{2}-1}}{(x+t-\eta)^{s}} d t=\int_{\frac{1}{2}-\eta}^{\infty} \frac{u^{s_{2}-1}}{(1+u)^{s}} d u \\
\leq \int_{0}^{\infty} \frac{u^{s_{2}-1}}{(1+u)^{s}} d u=B\left(s_{2}, s-s_{2}\right)=k_{s}\left(s_{2}\right) .
\end{gathered}
$$

On the other hand, in view of the decreasingness property of series, setting $u=\frac{t-\eta}{x}$, we obtain:

$$
\begin{aligned}
& \omega\left(s_{2}, x\right)=x^{s-s_{2}} \sum_{n=1}^{\infty} g(x, n)>x^{s-s_{2}} \int_{1}^{\infty} g(x, t) d t \\
& =\int_{\frac{1-\eta}{x}}^{\infty} \frac{u^{s_{2}-1}}{(1+u)^{s}} d u=B\left(s_{2}, s-s_{2}\right)-\int_{0}^{\frac{1-\eta}{x}} \frac{u^{s_{2}-1}}{(1+u)^{s}} d u \\
& =k_{s}\left(s_{2}\right)\left(1-O\left(\frac{1}{x^{s}}\right)\right)>0,
\end{aligned}
$$


where $O\left(\frac{1}{x^{s_{2}}}\right)=\frac{1}{k_{s}\left(s_{2}\right)} \int_{0}^{\frac{1-\eta}{x}} \frac{u^{s_{2}-1}}{(1+u)^{s}} d u>0$, which satisfies:

$$
0<\int_{0}^{\frac{1-\eta}{x}} \frac{u^{s_{2}-1}}{(1+u)^{s}} d u<\int_{0}^{\frac{1-\eta}{x}} u^{s_{2}-1} d u=\frac{1}{s_{2}}\left(\frac{1-\eta}{x}\right)^{s_{2}}\left(x \in \mathrm{R}_{+}\right) .
$$

In this case, we obtain (12).

(ii) For $s_{2} \in\left[1, \frac{3}{2}\right] \cap(0, s)$, by (9), we have:

$$
\begin{aligned}
\sum_{n=1}^{\infty} g(x, n) & =\int_{1}^{\infty} g(x, t) d t+\frac{1}{2} g(x, 1)+\int_{1}^{\infty} P_{1}(t) \frac{\partial}{\partial t} g(x, t) d t \\
& =\int_{\eta}^{\infty} g(x, t) d t-h(x),
\end{aligned}
$$

where $h(x)$ is defined by:

$$
h(x):=\int_{\eta}^{1} g(x, t) d t-\frac{1}{2} g(x, 1)-\int_{1}^{\infty} P_{1}(t) \frac{\partial}{\partial t} g(x, t) d t .
$$

We obtain $-\frac{1}{2} g(x, 1)=\frac{-(1-\eta)^{s_{2}-1}}{2(x+1-\eta)^{s}}$, and then integrating by parts, it follows that:

$$
\begin{aligned}
& \int_{\eta}^{1} g(x, t) d t=\int_{\eta}^{1} \frac{(t-\eta)^{s_{2}-1}}{(x+t-\eta)^{s}} d t=\frac{1}{s_{2}} \int_{\eta}^{1} \frac{d(t-\eta)^{s_{2}}}{(x+t-\eta)^{s}}=\left.\frac{1}{s_{2}} \frac{(t-\eta)^{s_{2}}}{(x+t-\eta)^{s}}\right|_{\eta} ^{1}+\frac{s}{s_{2}} \int_{\eta}^{1} \frac{(t-\eta)^{s_{2}} d t}{(x+t-\eta)^{s+1}} \\
&=\frac{1}{s_{2}} \frac{(1-\eta)^{s_{2}}}{(x+1-\eta)^{s}}+\frac{s}{s_{2}\left(s_{2}+1\right)} \int_{\eta}^{1} \frac{1}{(x+1-\eta)^{s+1}} d(t-\eta)^{s_{2}+1} \\
&>\frac{1}{s_{2}} \frac{(1-\eta)^{s_{2}}}{(x+1-\eta)^{s}}+\frac{s}{s_{2}\left(s_{2}+1\right)}\left[\frac{(t-\eta)^{s_{2}+1}}{(x+t-\eta)^{s+1}}\right]+\frac{1}{\eta}+\frac{s(s+1)}{s_{2}\left(s_{2}+1\right)(x+1-\eta)^{s+2}} \int_{\eta}^{1}(t-\eta)^{s_{2}+1} d t \\
&=\frac{1}{s_{2}} \frac{(1-\eta)^{s_{2}}}{(x+1-\eta)^{s}}+\frac{s}{s_{2}\left(s_{2}+1\right)} \frac{(1-\eta)^{s_{2}+1}}{(x+1-\eta)^{s+1}}+\frac{s(s+1)(1-\eta)^{s_{2}+2}}{s_{2}\left(s_{2}+1\right)\left(s_{2}+2\right)(x+1-\eta)^{s+2}} .
\end{aligned}
$$

We find:

$$
\begin{aligned}
-\frac{\partial}{\partial t} g(x, t) & =-\frac{\left(s_{2}-1\right)(t-\eta)^{s_{2}-2}}{(x+t-\eta)^{s}}+\frac{s(t-\eta)^{s_{2}-1}}{(x+t-\eta)^{s+1}} \\
& =\frac{\left(1-s_{2}\right)(t-\eta)^{s_{2}-2}}{(x+t-\eta)^{s}}+\frac{s(t-\eta)^{s_{2}-2}}{(x+t-\eta)^{s}}-\frac{s x(t-\eta)^{s_{2}-2}}{(x+t-\eta)^{s+1}} \\
& =\frac{\left(s+1-s_{2}\right)(t-\eta)^{s_{2}-2}}{(x+t-\eta)^{s}}-\frac{s x(t-\eta)^{s_{2}-2}}{(x+t-\eta)^{s+1}}
\end{aligned}
$$

additionally, for $s_{2} \in\left[1, \frac{3}{2}\right] \cap(0, s)$, it follows that:

$$
(-1)^{i} \frac{\partial^{i}}{\partial t^{i}}\left[\frac{(t-\eta)^{s_{2}-2}}{(x+t-\eta)^{s}}\right]>0,(-1)^{i} \frac{\partial^{i}}{\partial t^{i}}\left[\frac{(t-\eta)^{s_{2}-2}}{(x+t-\eta)^{s+1}}\right]>0(t>\eta ; i=0,1,2,3) .
$$

By (8), (9) and (10), setting $a:=1-\eta\left(\in\left[\frac{3}{4}, 1\right]\right)$, we obtain:

$$
\begin{aligned}
& \left(s+1-s_{2}\right) \int_{1}^{\infty} P_{1}(t) \frac{(t-\eta)^{s_{2}-2}}{(x+t-\eta)^{s}} d t>-\frac{s+1-s_{2}}{12(x+1-\eta)^{s}} a^{s_{2}-2} \\
& -x s \int_{1}^{\infty} P_{1}(t) \frac{(t-\eta)^{s_{2}-2}}{(x+t-\eta)^{s+1}} d t>\frac{x s}{12(x+1-\eta)^{s+1}} a^{s_{2}-2}-\frac{x s}{720}\left[\frac{(t-\eta)^{s_{2}-2}}{(x+t-\eta)^{s+1}}\right]_{t=1}^{\prime \prime} \\
& >\frac{(x+1-\eta) s-a s}{12(x+1-\eta)^{s+1}} a^{s_{2}-2}-\frac{(x+1-\eta) s}{720}\left[\frac{(s+1)(s+2) a^{s_{2}-2}}{(x+1-\eta)^{s+3}}+\frac{2(s+1)\left(2-s_{2}\right) a^{s_{2}-3}}{(x+1-\eta)^{s+2}}+\frac{\left(2-s_{2}\right)\left(3-s_{2}\right) a^{s_{2}-4}}{(x+1-\eta)^{s+1}}\right] . \\
& =\frac{s a^{s_{2}-2}}{12(x+1-\eta)^{s}}-\frac{s a^{s_{2}-1}}{12(x+1-\eta)^{s+1}}-\frac{s}{720}\left[\frac{(s+1)(s+2) a^{s_{2}-2}}{(x+1-\eta)^{s+2}}+\frac{2(s+1)\left(2-s_{2}\right) a^{s_{2}-3}}{(x+1-\eta)^{s+1}}+\frac{\left(2-s_{2}\right)\left(3-s_{2}\right) a^{s_{2}-4}}{(x+1-\eta)^{s}}\right],
\end{aligned}
$$


and then we have:

$$
h(x)>\frac{a^{s_{2}-4}}{(x+1-\eta)^{s}} h_{1}+\frac{s a^{s_{2}-3}}{(x+1-\eta)^{s+1}} h_{2}+\frac{s(s+1) a^{s_{2}-2}}{(x+1-\eta)^{s+2}} h_{3},
$$

where $h_{i}(i=1,2,3)$ are indicated as:

$$
\begin{gathered}
h_{1}:=\frac{a^{4}}{s_{2}}-\frac{a^{3}}{2}-\frac{\left(1-s_{2}\right) a^{2}}{12}-\frac{s\left(2-s_{2}\right)\left(3-s_{2}\right)}{720}, \\
h_{2}:=\frac{a^{4}}{s_{2}\left(s_{2}+1\right)}-\frac{a^{2}}{12}-\frac{(s+1)\left(2-s_{2}\right)}{360}, \\
h_{3}:=\frac{a^{4}}{s_{2}\left(s_{2}+1\right)\left(s_{2}+2\right)}-\frac{s+2}{720} .
\end{gathered}
$$

For $s \in(0,4], s_{2} \in\left[1, \frac{3}{2}\right] \cap(0, s), a \in\left[\frac{3}{4}, 1\right]$, we find:

$$
h_{1}>\frac{a^{2}}{12 s_{2}}\left[s_{2}^{2}-(6 a+1) s_{2}+12 a^{2}\right]-\frac{1}{90} .
$$

In view of:

$$
\begin{gathered}
\frac{d}{d a}\left[s_{2}^{2}-(6 a+1) s_{2}+12 a^{2}\right]=6\left(4 a-s_{2}\right) \geq 6\left(4 \cdot \frac{3}{4}-\frac{3}{2}\right)>0, \text { and } \\
\frac{d}{d s_{2}}\left[s_{2}^{2}-(6 a+1) s_{2}+12 a^{2}\right]=2 s_{2}-(6 a+1) \\
\leq 2 \cdot \frac{3}{2}-\left(6 \cdot \frac{3}{4}+1\right)=3-\frac{11}{2}<0,
\end{gathered}
$$

we obtain:

$$
\begin{gathered}
h_{1} \geq \frac{(3 / 4)^{2}}{12(3 / 2)}\left[\left(\frac{3}{2}\right)^{2}-\left(6 \cdot \frac{3}{4}+1\right) \frac{3}{2}+12\left(\frac{3}{4}\right)^{2}\right]-\frac{1}{90} \\
h_{2}>a^{2}\left(\frac{4 a^{2}}{15}-\frac{1}{12}\right)-\frac{1}{72} \geq\left(\frac{3}{4}\right)^{2}\left[\frac{4}{15}\left(\frac{3}{4}\right)^{2}-\frac{1}{12}\right]-\frac{1}{72}=\frac{3}{80}-\frac{1}{72}>0, \\
h_{3} \geq \frac{8 a^{4}}{105}-\frac{6}{720} \geq \frac{8}{105}\left(\frac{3}{4}\right)^{4}-\frac{1}{120}=\frac{27}{1120}-\frac{1}{120}>0,
\end{gathered}
$$

and then we obtain $h(x)>0$.

On the other hand, similar to the above, we have:

$$
\begin{aligned}
\sum_{n=1}^{\infty} g(x, n) & =\int_{1}^{\infty} g(x, t) d t+\frac{1}{2} g(x, 1)+\int_{1}^{\infty} P_{1}(t) \frac{\partial}{\partial t} g(x, t) d t \\
& =\int_{1}^{\infty} g(x, t) d t+H(x),
\end{aligned}
$$

where $H(x)$ is indicated as:

$$
H(x):=\frac{1}{2} g(x, 1)+\int_{1}^{\infty} P_{1}(t) \frac{\partial}{\partial t} g(x, t) d t .
$$

Thus, we obtain that $\frac{1}{2} g(x, 1)=\frac{a^{s_{2}-1}}{2(x+1-\eta)^{s}}$ and:

$$
\frac{\partial}{\partial t} g(x, t)=-\frac{\left(s+1-s_{2}\right)(t-\eta)^{s_{2}-2}}{(x+t-\eta)^{s}}+\frac{s x(t-\eta)^{s_{2}-2}}{(x+t-\eta)^{s+1}} .
$$

For $s_{2} \in\left(0, \frac{3}{2}\right] \cap(0, s), 0<s \leq 4$, by (7), we obtain:

$$
-\left(s+1-s_{2}\right) \int_{1}^{\infty} P_{1}(t) \frac{(t-\eta)^{s_{2}-2}}{(x+t-\eta)^{s}} d t>0
$$




$$
\begin{aligned}
& x s \int_{1}^{\infty} P_{1}(t) \frac{(t-\eta)^{s_{2}-2}}{(x+t-\eta)^{s+1}} d t>\frac{-x s}{12(x+1-\eta)^{s+1}} a^{s_{2}-2}=\frac{-(x+1-\eta) s+a s}{12(x+1-\eta)^{s+1}} a^{s_{2}-2} \\
& =\frac{-s}{12(x+1-\eta)^{s}} a^{s_{2}-2}+\frac{s}{12(x+1-\eta)^{s+1}} a^{s_{2}-1}>\frac{-s}{12(x+1-\eta)^{s}} a^{s_{2}-2} .
\end{aligned}
$$

Hence, we have:

$$
\begin{aligned}
H(x) & >\frac{a^{s_{2}-1}}{2(x+1-\eta)^{s}}-\frac{s a^{s_{2}-2}}{12(x+1-\eta)^{s}}=\left(\frac{a}{2}-\frac{s}{12}\right) \frac{a^{s_{2}-2}}{(x+1-\eta)^{s}} \\
& \geq\left(\frac{1}{2} \cdot \frac{3}{4}-\frac{4}{12}\right) \frac{a^{s_{2}-2}}{(x+1-\eta)^{s}}=\left(\frac{3}{8}-\frac{1}{3}\right) \frac{a^{s_{2}-2}}{(x+1-\eta)^{s}}>0 .
\end{aligned}
$$

Therefore, we obtain:

$$
\int_{1}^{\infty} g(x, t) d t<\sum_{n=1}^{\infty} g(x, n)<\int_{\eta}^{\infty} g(x, t) d t(x>0)
$$

In view of the results obtained in the case (i), we obtain (12). This completes the proof of lemma 2.

Lemma 3. Let $s \in(0,4], s_{1} \in(0, s), s_{2} \in\left(0, \frac{3}{2}\right] \cap(0, s)$.Then, we have the following more accurate half-discrete Hardy-Hilbert inequality:

$$
\begin{gathered}
I=\int_{0}^{\infty} \sum_{n=1}^{\infty} \frac{a_{n} f(x)}{(x+n-\eta)^{s}} d x \leq\left(k_{s}\left(s_{2}\right)\right)^{\frac{1}{p}}\left(k_{s}\left(s_{1}\right)\right)^{\frac{1}{q}} \\
\times\left\{\int_{0}^{\infty} x^{p\left[1-\left(\frac{s-s_{2}}{p}+\frac{s_{1}}{q}\right)\right]-1} f^{p}(x) d x\right\}^{\frac{1}{p}}\left\{\sum_{n=1}^{\infty}(n-\eta)^{q\left[1-\left(\frac{s-s_{1}}{q}+\frac{s_{2}}{p}\right)\right]-1} a_{n}^{q}\right\}^{\frac{1}{q}} .
\end{gathered}
$$

Proof. For $s_{1} \in(0, s)$, setting $u=\frac{x}{n-\eta}$, we have the following expression of the weight coefficient:

$$
\omega\left(s_{1}, n\right):=(n-\eta)^{s-s_{1}} \int_{0}^{\infty} \frac{x^{s_{1}-1}}{(x+n-\eta)^{s}} d x=\int_{0}^{\infty} \frac{u^{s_{1}-1}}{(u+1)^{s}} d u=k_{s}\left(s_{1}\right)(n \in \mathrm{N}) .
$$

By using Hölder's inequality [18], we obtain:

$$
\begin{aligned}
I= & \int_{0}^{\infty} \sum_{n=1}^{\infty} \frac{1}{(x+n-\eta)^{s}}\left[\frac{x^{(1-s) 1 / q}}{(n-\eta)^{\left(1-s_{2}\right) / p}} f(x)\right]\left[\frac{(n-\eta)^{\left(1-s_{2}\right) / p}}{x^{(1-s) 1 / q}} a_{n}\right] d x \\
\leq & {\left[\int_{0}^{\infty} \sum_{n=1}^{\infty} \frac{1}{(x+n-\eta)^{s}} \frac{x^{(1-s 1)(p-1)}}{(n-\eta)^{1-s_{2}}} f^{p}(x) d x\right] \frac{1}{p} } \\
& \times\left[\sum_{n=1}^{\infty} \int_{0}^{\infty} \frac{1}{(x+n-\eta)^{s}} \frac{(n-\eta)^{\left(1-s_{2}\right)(q-1)}}{x^{1-s 1}} d x a_{n}^{q}\right]^{\frac{1}{q}} \\
= & \left\{\int_{0}^{\infty} \omega\left(s_{2}, x\right) x^{p\left[1-\left(\frac{s-s_{2}}{p}+\frac{s 1}{q}\right)\right]-1} f^{p}(x) d x\right\} \frac{1}{p} \\
& \times\left\{\sum_{n=1}^{\infty} \omega(s, 1 n)(n-\eta)^{q\left[1-\left(\frac{s-s_{1}}{q}+\frac{s_{2}}{p}\right)\right]-1} a_{n}^{q}\right\} \frac{1}{q} .
\end{aligned}
$$

Then, by (12) and (14), we derive inequality (13). The Lemma 3 is proved.

Remark 1. In (13), for $s=\lambda+2 \in(2,4], \lambda \in(0,2], s_{1}=\lambda_{1}+1 \in(1, s), \lambda_{1} \in(0, \lambda+1)$,

$$
s_{2}=\lambda_{2}+1 \in\left(1, \frac{3}{2}\right], \lambda_{2} \in\left(0, \frac{1}{2}\right] \cap(0, \lambda+1)
$$


Replacing $f(x)\left(\right.$ resp. $\left.a_{n}\right) b y F(x)\left(\right.$ resp. $\left.A_{n}\right)$, in view of Lemma 3 and (5), we have:

$$
\begin{gathered}
\int_{0}^{\infty} \sum_{n=1}^{\infty} \frac{A_{n}}{(x+n-\eta)^{\lambda+2}} F(x) d x<\left(k_{\lambda+2}\left(\lambda_{2}+1\right)\right)^{\frac{1}{p}}\left(k_{\lambda+2}\left(\lambda_{1}+1\right)\right)^{\frac{1}{q}} \\
\times\left[\int_{0}^{\infty} x^{-p \hat{\lambda}_{1}-1} F^{p}(x) d x\right]^{\frac{1}{p}}\left[\sum_{n=1}^{\infty}(n-\eta)^{-q \hat{\lambda}_{2}-1} A_{n}^{q}\right]^{\frac{1}{q}} .
\end{gathered}
$$

Lemma 4. For $t>0$, we have:

$$
\begin{aligned}
& \int_{0}^{\infty} e^{-t x} f(x) d x=t \int_{0}^{\infty} e^{-t x} F(x) d x \\
& \sum_{n=1}^{\infty} e^{-t(n-\eta)} a_{n} \leq t \sum_{n=1}^{\infty} e^{-t(n-\eta)} A_{n} .
\end{aligned}
$$

Proof. Integration by parts, in view of $F(0)=0, F(x)=o\left(e^{t x}\right)(t>0 ; x \rightarrow \infty)$, it follows that:

$$
\begin{gathered}
\int_{0}^{\infty} \sum_{n=1}^{\infty} \frac{A_{n}}{(x+n-\eta)^{\lambda+2}} F(x) d x<\left(k_{\lambda+2}\left(\lambda_{2}+1\right)\right)^{\frac{1}{p}}\left(k_{\lambda+2}\left(\lambda_{1}+1\right)\right)^{\frac{1}{q}} \\
=\lim _{x \rightarrow \infty} e^{-t x} F(x)+t \int_{0}^{\infty} e^{-t x} F(x) d x=t \int_{0}^{\infty} e^{-t x} F(x) d x
\end{gathered}
$$

and then (16) follows.

In view of $A_{n} e^{-t(n-\eta)}=o(1)(n \rightarrow \infty)$, by Abel's summation by parts formula, we obtain:

$$
\begin{array}{r}
\sum_{n=1}^{\infty} e^{-t(n-\eta)} a_{n}=\lim _{n \rightarrow \infty} A_{n} e^{-t(n-\eta)}+\sum_{n=1}^{\infty} A_{n}\left[e^{-t(n-\eta)}-e^{-t(n-\eta+1)}\right] \\
=\sum_{n=1}^{\infty} A_{n}\left[e^{-t(n-\eta)}-e^{-t(n-\eta+1)}\right]=\left(1-e^{-t}\right) \sum_{n=1}^{\infty} e^{-t(n-\eta)} A_{n} .
\end{array}
$$

Since $1-e^{-t}<t(t>0)$, we have (17). The Lemma 4 is proved.

\section{Main Results}

Theorem 1. Let $p>1, \frac{1}{p}+\frac{1}{q}=1, \eta \in\left[0, \frac{1}{4}\right], \lambda \in(0,2], \lambda_{1} \in(0, \lambda+1), \lambda_{2} \in\left(0, \frac{1}{2}\right] \cap(0, \lambda+1)$, $\hat{\lambda}_{1}:=\frac{\lambda-\lambda_{2}}{p}+\frac{\lambda_{1}}{q}, \hat{\lambda}_{2}:=\frac{\lambda-\lambda_{1}}{q}+\frac{\lambda_{2}}{p}$. Then, we have the following half-discrete Hilbert-type inequality:

$$
\begin{aligned}
I:=\int_{0}^{\infty} \sum_{n=1}^{\infty} \frac{a_{n}}{(x+n-\eta)^{\lambda}} f(x) d x<\frac{\Gamma(\lambda+2)}{\Gamma(\lambda)}\left(k_{\lambda+2}\left(\lambda_{2}+1\right)\right)^{\frac{1}{p}}\left(k_{\lambda+2}\left(\lambda_{1}+1\right)\right)^{\frac{1}{q}} \\
\times\left[\int_{0}^{\infty} x^{-p \hat{\lambda}_{1}-1} F^{p}(x) d x\right]^{\frac{1}{p}}\left[\sum_{n=1}^{\infty}(n-\eta)^{-q \hat{\lambda} 2-1} A_{n}^{q}\right]^{\frac{1}{q}} .
\end{aligned}
$$

In particular, for $\lambda_{1}+\lambda_{2}=\lambda(\in(0,2])\left(\lambda_{1} \in(0, \lambda), \lambda_{2} \in\left(0, \frac{1}{2}\right] \cap(0, \lambda)\right)$, we have:

$$
\begin{gathered}
\int_{0}^{\infty} \sum_{n=1}^{\infty} \frac{a_{n}}{(x+n-\eta)^{\lambda}} f(x) d x<\lambda_{1} \lambda_{2} B\left(\lambda_{1}, \lambda_{2}\right) \\
\times\left[\int_{0}^{\infty} x^{-p \lambda_{1}-1} F^{p}(x) d x\right]^{\frac{1}{p}}\left[\sum_{n=1}^{\infty}(n-\eta)^{-q \lambda_{2}-1} A_{n}^{q}\right]^{\frac{1}{q}} .
\end{gathered}
$$


Proof. Since for $\lambda>0$, we have:

$$
\frac{1}{(x+n-\eta)^{\lambda}}=\frac{1}{\Gamma(\lambda)} \int_{0}^{\infty} t^{\lambda-1} e^{-(x+n-\eta) t} d t
$$

it follows that:

$$
\begin{aligned}
I & =\frac{1}{\Gamma(\lambda)} \int_{0}^{\infty} \sum_{n=1}^{\infty} a_{n} f(x) \int_{0}^{\infty} t^{\lambda-1} e^{-(x+n-\eta) t} d t d x \\
& =\frac{1}{\Gamma(\lambda)} \int_{0}^{\infty} t^{\lambda-1} \int_{0}^{\infty} e^{-x t} f(x) d x \sum_{n=1}^{\infty} e^{-(n-\eta) t} a_{n} d t \\
& \leq \frac{1}{\Gamma(\lambda)} \int_{0}^{\infty} t^{\lambda+1} \int_{0}^{\infty} e^{-x t} F(x) d x \sum_{n=1}^{\infty} e^{-(n-\eta) t} A_{n} d t \\
= & \frac{1}{\Gamma(\lambda)} \int_{0}^{\infty} \sum_{n=1}^{\infty} A_{n} F(x) \int_{0}^{\infty} t^{(\lambda+2)-1} e^{-(x+n-\eta) t} d t d x \\
& =\frac{\Gamma(\lambda+2)}{\Gamma(\lambda)} \int_{0}^{\infty} \sum_{n=1}^{\infty} \frac{A_{n}}{(x+n-\eta)^{\lambda+2}} F(x) d x
\end{aligned}
$$

By applying (15), we obtain (18).

In particular, for $\lambda_{1}+\lambda_{2}=\lambda(\in(0,2])\left(\lambda_{1} \in(0, \lambda), \lambda_{2} \in\left(0, \frac{1}{2}\right] \cap(0, \lambda)\right)$, one has:

$$
\begin{gathered}
k_{\lambda+2}\left(\lambda_{2}+1\right)=k_{\lambda+2}\left(\lambda_{1}+1\right)=B\left(\lambda_{1}+1, \lambda_{2}+1\right) \\
=\frac{\Gamma\left(\lambda_{1}+1\right) \Gamma\left(\lambda_{2}+1\right)}{\Gamma(\lambda+2)}=\frac{\lambda_{1} \lambda_{2} \Gamma\left(\lambda_{1}\right) \Gamma\left(\lambda_{2}\right)}{\Gamma(\lambda+2)}=\frac{\Gamma(\lambda)}{\Gamma(\lambda+2)} \lambda_{1} \lambda_{2} B\left(\lambda_{1}, \lambda_{2}\right) .
\end{gathered}
$$

Hence, it follows from (18) that:

$$
\begin{gathered}
\int_{0}^{\infty} \sum_{n=1}^{\infty} \frac{a_{n}}{(x+n-\eta)^{\lambda}} f(x) d x<\lambda_{1} \lambda_{2} B\left(\lambda_{1}, \lambda_{2}\right) \\
\times\left[\int_{0}^{\infty} x^{-p \lambda_{1}-1} F^{p}(x) d x\right]^{\frac{1}{p}}\left[\sum_{n=1}^{\infty}(n-\eta)^{-q \lambda_{2}-1} A_{n}^{q}\right]^{\frac{1}{q}},
\end{gathered}
$$

which is the desired inequality (19).

Remark 3. Putting $\eta=0$ in (20), we have:

$$
\begin{aligned}
& \int_{0}^{\infty} \sum_{n=1}^{\infty} \frac{a_{n}}{(x+n)^{\lambda}} f(x) d x<\lambda_{1} \lambda_{2} B\left(\lambda_{1}, \lambda_{2}\right) \\
& \times\left[\int_{0}^{\infty} x^{-p \lambda_{1}-1} F^{p}(x) d x\right]^{\frac{1}{p}}\left[\sum_{n=1}^{\infty} n^{-q \lambda_{2}-1} A_{n}^{q}\right]^{\frac{1}{q}} .
\end{aligned}
$$

Namely, (18) given by Theorem 1 is a more accurate extension of (21) above. It should be noted that here the statement of "more accurate inequality" borrows from the statement mentioned at the beginning of the paper on the comparison between inequalities (1) and (2) described in the previous literature.

Theorem 2. If $\lambda-\lambda_{1} \leq \frac{1}{2}$, then the following statements (i), (ii) and (iii), associated with Theorem 1 , are equivalent:

(i) $\quad\left(k_{\lambda+2}\left(\lambda_{2}+1\right)\right)^{\frac{1}{p}}\left(k_{\lambda+2}\left(\lambda_{1}+1\right)\right)^{\frac{1}{q}} \leq k_{\lambda+2}\left(\frac{\lambda-\lambda_{2}}{p}+\frac{\lambda_{1}}{q}+1\right)$;

(ii) $\lambda_{1}+\lambda_{2}=\lambda(\in(0,2])$, where $\lambda_{1} \in(0, \lambda), \lambda_{2} \in\left(0, \frac{1}{2}\right] \cap(0, \lambda)$;

(iii) The constant factor:

$$
\frac{\Gamma(\lambda+2)}{\Gamma(\lambda)}\left(k_{\lambda+2}\left(\lambda_{2}+1\right)\right)^{\frac{1}{p}}\left(k_{\lambda+2}\left(\lambda_{1}+1\right)\right)^{\frac{1}{q}}
$$


in (19) is the best possible.

Proof. “ (i) $\Rightarrow$ (ii)”. By using Hölder inequality with weight, we obtain:

$$
\begin{gathered}
k_{\lambda+2}\left(\frac{\lambda-\lambda_{2}}{p}+\frac{\lambda_{1}}{q}+1\right) \\
=\int_{0}^{\infty} \frac{1}{(1+u)^{\lambda+2}} u^{\frac{\lambda-\lambda_{2}}{p}+\frac{\lambda_{1}}{q}} d u=\int_{0}^{\infty} \frac{1}{(1+u)^{\lambda+2}}\left(u^{\frac{\lambda-\lambda_{2}}{p}}\right)\left(u^{\frac{\lambda_{1}}{q}}\right) d u \\
\leq\left[\int_{0}^{\infty} \frac{1}{(1+u)^{\lambda+2}} u^{\lambda-\lambda_{2}} d u\right]^{\frac{1}{p}}\left[\int_{0}^{\infty} \frac{1}{(1+u)^{\lambda+2}} u^{\lambda_{1}} d u\right]^{\frac{1}{q}} \\
=\left[\int_{0}^{\infty} \frac{1}{(1+v)^{\lambda+2}} v^{\left(\lambda_{2}+1\right)-1} d v\right]^{\frac{1}{p}}\left[\int_{0}^{\infty} \frac{1}{(1+u)^{\lambda+2}} u^{\left(\lambda_{1}+1\right)-1} d u\right]^{\frac{1}{q}} \\
=\left(k_{\lambda+2}\left(\lambda_{2}+1\right)\right)^{\frac{1}{p}}\left(k_{\lambda+2}\left(\lambda_{1}+1\right)\right)^{\frac{1}{q}} .
\end{gathered}
$$

In view of inequality (i), we conclude that (22) keeps the form of equality.

We observe that (22) keeps the form of equality if and only if there exist constants $A$ and $B$, such that they are not both zero and $A u^{\lambda-\lambda_{2}}=B u^{\lambda_{1}}$ a.e. in $\mathrm{R}_{+}$(see [18]). Assuming that $A \neq 0$, we have $u^{\lambda-\lambda_{2}-\lambda_{1}}=\frac{B}{A}$ a.e. in $\mathrm{R}_{+}$, and then $\lambda-\lambda_{2}-\lambda_{1}=0$, namely, $\lambda_{1}+\lambda_{2}=$ $\lambda(\in(0,2])$, where, $\lambda_{1} \in(0, \lambda), \lambda_{2} \in\left(0, \frac{1}{2}\right] \cap(0, \lambda)$.

“(ii) $\Rightarrow$ (iii)". For $\lambda_{1}+\lambda_{2}=\lambda(\in(0,2]), \lambda_{1} \in(0, \lambda), \lambda_{2} \in\left(0, \frac{1}{2}\right] \cap(0, \lambda),(19)$ reduces to (20). For any $0<\varepsilon<\min \left\{p \lambda_{1}, q \lambda_{2}\right\}$, we set:

$$
\widetilde{f}(x):=\left\{\begin{array}{c}
0,0<x<1, \\
x^{\lambda_{1}-\frac{\varepsilon}{p}-1}, x \geq 1
\end{array}, \widetilde{a}_{n}:=n^{\lambda_{2}-\frac{\varepsilon}{q}-1}(n \in \mathrm{N})\right.
$$

Then, it follows that:

$$
\begin{aligned}
& \widetilde{F}(x):=\int_{0}^{x} \widetilde{f}(t) d t \leq\left\{\begin{array}{l}
0,0<x<1, \\
\frac{1}{\lambda_{1}-\frac{\varepsilon}{p}} x^{\lambda_{1}-\frac{\varepsilon}{p}}, x \geq 1
\end{array}\right. \\
& \widetilde{A}_{n}:=\sum_{k=1}^{n} \widetilde{a}_{k}=\sum_{k=1}^{n} k^{\lambda_{2}-\frac{\varepsilon}{q}-1}<\int_{0}^{n} t^{\lambda_{2}-\frac{\varepsilon}{q}-1} d t=\frac{1}{\lambda_{2}-\frac{\varepsilon}{q}} n^{\lambda_{2}-\frac{\varepsilon}{q}}(n \in \mathrm{N}) .
\end{aligned}
$$

If there exists a positive constant $M \leq \lambda_{1} \lambda_{2} B\left(\lambda_{1}, \lambda_{2}\right)$ such that (20) is valid when replacing $\lambda_{1} \lambda_{2} B\left(\lambda_{1}, \lambda_{2}\right)$ by $M$, then, in particular for $\eta=0$, by substitution of $f(x)=\widetilde{f}(x), a_{n}=\widetilde{a}_{n}$, $F(x)=\widetilde{F}(x)$ and $A_{n}=\widetilde{A}_{n}$ in (21), we have:

$$
\widetilde{I}:=\int_{0}^{\infty} \sum_{n=1}^{\infty} \frac{\widetilde{a}_{n} \widetilde{f}(x)}{(x+n)^{\lambda}} d x<M\left(\int_{0}^{\infty} x^{-p \lambda_{1}-1} \widetilde{F}^{p}(x) d x\right)^{\frac{1}{p}}\left(\sum_{n=1}^{\infty} n^{-q \lambda_{2}-1} \widetilde{A}_{n}^{q}\right)^{\frac{1}{q}} .
$$

In the following, we show that $\lambda_{1} \lambda_{2} B\left(\lambda_{1}, \lambda_{2}\right) \leq M$, and then $M=\lambda_{1} \lambda_{2} B\left(\lambda_{1}, \lambda_{2}\right)$ is the best possible constant factor in (20).

By (23) and the decreasingness property of series, we obtain:

$$
\begin{gathered}
\widetilde{I}<M \frac{1}{\lambda_{1}-\frac{\varepsilon}{p}}\left(\int_{1}^{\infty} x^{-p \lambda_{1}-1} x^{p \lambda_{1}-\varepsilon} d x\right)^{\frac{1}{p}} \frac{1}{\lambda_{2}-\frac{\varepsilon}{q}}\left(\sum_{n=1}^{\infty} n^{-q \lambda_{2}-1} n^{q \lambda_{2}-\varepsilon}\right)^{\frac{1}{q}} \\
=M\left(\frac{1}{\lambda_{1}-\frac{\varepsilon}{p}}\right)\left(\frac{1}{\lambda_{2}-\frac{\varepsilon}{q}}\right)\left(\int_{1}^{\infty} x^{-\varepsilon-1} d x\right)^{\frac{1}{p}}\left(1+\sum_{n=2}^{\infty} n^{-\varepsilon-1}\right)^{\frac{1}{q}} \\
<M\left(\frac{1}{\lambda_{1}-\frac{\varepsilon}{p}}\right)\left(\frac{1}{\lambda_{2}-\frac{\varepsilon}{q}}\right)\left(\int_{1}^{\infty} x^{-\varepsilon-1} d x\right)^{\frac{1}{p}}\left(1+\int_{1}^{\infty} y^{-\varepsilon-1} d y\right)^{\frac{1}{q}} \\
=\frac{M}{\varepsilon}\left(\frac{1}{\lambda_{1}-\frac{\varepsilon}{p}}\right)\left(\frac{1}{\lambda_{2}-\frac{\varepsilon}{q}}\right)(\varepsilon+1)^{\frac{1}{q}} .
\end{gathered}
$$


By (11) (for $\eta=0)$, setting $\widetilde{\lambda}_{2}=\lambda_{2}-\frac{\varepsilon}{q} \in\left(0, \frac{1}{2}\right) \cap(0, \lambda)\left(0<\widetilde{\lambda}_{1}=\lambda_{1}+\frac{\varepsilon}{q}<\lambda\right)$, we obtain:

$$
\begin{aligned}
\widetilde{I} & =\int_{1}^{\infty}\left[x^{\left(\lambda_{1}+\frac{\varepsilon}{q}\right)} \sum_{n=1}^{\infty} \frac{1}{(x+n)^{\lambda}} n^{\left(\lambda_{2}-\frac{\varepsilon}{q}\right)-1}\right] x^{-\varepsilon-1} d x \\
& =\int_{1}^{\infty} \omega\left(\widetilde{\lambda}_{2}, x\right) x^{-\varepsilon-1} d x>B\left(\widetilde{\lambda}_{1}, \widetilde{\lambda}_{2}\right) \int_{1}^{\infty}\left[1-O\left(\frac{1}{x^{\lambda_{2}}}\right)\right] x^{-\varepsilon-1} d x \\
& =B\left(\widetilde{\lambda}_{1}, \widetilde{\lambda}_{2}\right)\left[\int_{1}^{\infty} x^{-\varepsilon-1} d x-\int_{1}^{\infty} O\left(\frac{1}{x^{\lambda_{2}+\frac{\varepsilon}{p}+1}}\right) d x\right] \\
& =\frac{1}{\varepsilon} B\left(\lambda_{1}+\frac{\varepsilon}{q}, \lambda_{2}-\frac{\varepsilon}{q}\right)(1-\varepsilon O(1)) .
\end{aligned}
$$

Then, in virtue of the above results, we have:

$$
B\left(\lambda_{1}+\frac{\varepsilon}{q}, \lambda_{2}-\frac{\varepsilon}{q}\right)(1-\varepsilon O(1))<\varepsilon \widetilde{I}<M\left(\frac{1}{\lambda_{1}-\frac{\varepsilon}{p}}\right)\left(\frac{1}{\lambda_{2}-\frac{\varepsilon}{q}}\right)(\varepsilon+1)^{\frac{1}{q}} .
$$

Putting $\varepsilon \rightarrow 0^{+}$, in view of the continuity of the beta function, we obtain $\lambda_{1} \lambda_{2} B\left(\lambda_{1}, \lambda_{2}\right) \leq$ $M$. Hence, $M=\lambda_{1} \lambda_{2} B\left(\lambda_{1}, \lambda_{2}\right)$ is the best possible constant factor in (20).

“(iii) $\Rightarrow\left(\right.$ i)". Since $\lambda-\lambda_{1} \leq \frac{1}{2}$, for $\hat{\lambda}_{1}=\frac{\lambda-\lambda_{2}}{p}+\frac{\lambda_{1}}{q}, \hat{\lambda}_{2}=\frac{\lambda-\lambda_{1}}{q}+\frac{\lambda_{2}}{p}$, we find:

$$
\hat{\lambda}_{1}+\hat{\lambda}_{2}=\frac{\lambda-\lambda_{2}}{p}+\frac{\lambda_{1}}{q}+\frac{\lambda-\lambda_{1}}{q}+\frac{\lambda_{2}}{p}=\lambda, 0<\hat{\lambda}_{1}, \hat{\lambda}_{2}<\frac{\lambda}{p}+\frac{\lambda}{q}=\lambda,
$$

$\hat{\lambda}_{2} \leq \frac{1 / 2}{p}+\frac{1 / 2}{q}=\frac{1}{2}$, and $\hat{\lambda}_{1} \hat{\lambda}_{2} B\left(\hat{\lambda}_{1}, \hat{\lambda}_{2}\right) \in \mathrm{R}_{+}$.

If the constant factor $\frac{\Gamma(\lambda+2)}{\Gamma(\lambda)}\left(k_{\lambda+2}\left(\lambda_{2}+1\right)\right)^{\frac{1}{p}}\left(k_{\lambda+2}\left(\lambda_{1}+1\right)\right)^{\frac{1}{q}}$ in (19) is the best possible, then by (21) (for $\lambda_{i}=\hat{\lambda}_{i}(i=1,2)$ ), we have:

$$
\begin{aligned}
& \frac{\Gamma(\lambda+2)}{\Gamma(\lambda)}\left(k_{\lambda+2}\left(\lambda_{2}+1\right)\right)^{\frac{1}{p}}\left(k_{\lambda+2}\left(\lambda_{1}+1\right)\right)^{\frac{1}{q}} \\
& \leq \hat{\lambda}_{1} \hat{\lambda}_{2} B\left(\hat{\lambda}_{1}, \hat{\lambda}_{2}\right)=\frac{\Gamma(\lambda+2)}{\Gamma(\lambda)} k_{\lambda+2}\left(\hat{\lambda}_{1}+1\right) \\
& \quad=\frac{\Gamma(\lambda+2)}{\Gamma(\lambda)} k_{\lambda+2}\left(\frac{\lambda-\lambda_{2}}{p}+\frac{\lambda_{1}}{q}+1\right)\left(\in \mathrm{R}_{+}\right),
\end{aligned}
$$

namely, statement (i) is valid.

Hence, the statements (i), (ii) and (iii) are equivalent. This completes the proof of Theorem 2.

Remark 4. Putting $\eta=\frac{1}{4}$ in (20), we acquire:

$$
\begin{gathered}
\int_{0}^{\infty} \sum_{n=1}^{\infty} \frac{a_{n}}{\left(x+n-\frac{1}{4}\right)^{\lambda}} f(x) d x<\lambda_{1} \lambda_{2} B\left(\lambda_{1}, \lambda_{2}\right) \\
\times\left[\int_{0}^{\infty} x^{-p \lambda_{1}-1} F^{p}(x) d x\right]^{\frac{1}{p}}\left[\sum_{n=1}^{\infty}\left(n-\frac{1}{4}\right)^{-q \lambda_{2}-1} A_{n}^{q}\right]^{\frac{1}{q}}
\end{gathered}
$$

In particular, for $\lambda=1, \lambda_{1}=\lambda_{2}=\frac{1}{2}$, we have the following Hilbert-type inequality with the best possible constant factor $\frac{\pi}{4}$

$$
\int_{0}^{\infty} \sum_{n=1}^{\infty} \frac{a_{n}}{x+n-\frac{1}{4}} f(x) d x<\frac{\pi}{4}\left[\int_{0}^{\infty} x^{-\frac{p}{2}-1} F^{p}(x) d x\right]^{\frac{1}{p}}\left[\sum_{n=1}^{\infty}\left(n-\frac{1}{4}\right)^{-\frac{q}{2}-1} A_{n}^{q}\right]^{\frac{1}{q}} .
$$




\section{Conclusions}

In this paper, based on the weight coefficients and the idea of introducing parameters, by applying Hermite-Hadamard inequality, the Euler-Maclaurin summation formula and Abel's summation by parts formula, a more accurate half-discrete Hilbert-type inequality involving one upper limit function as well as one partial sum is given in Theorem 1 . The equivalent statements of the best possible constant factor related to several parameters are considered in Theorem 2. As applications of the main results, some new inequalities are proposed in Remarks 3 and 4. Our results would provide a significant supplement to the study of half-discrete Hilbert-type inequality.

Author Contributions: B.Y. carried out the mathematical studies, participated in the sequence alignment and drafted the manuscript. S.W. and X.H. participated in the design of the study and performed the numerical analysis. All authors contributed equally to the preparation of this paper. All authors have read and agreed to the published version of the manuscript.

Funding: This work is supported by the National Natural Science Foundation (No. 61772140, No.12071491), the Characteristic Innovation Project of Guangdong Provincial Colleges and Universities (No.2020KTSCX088), the Construction Project of Teaching Quality and Teaching Reform in Guangdong Undergraduate Colleges and Universities in 2018 (Speciality of Financial Mathematics), and the Natural Science Foundation of Fujian Province of China (No. 2020J01365).

Institutional Review Board Statement: Not applicable.

Informed Consent Statement: Not applicable.

Data Availability Statement: The data used to support the findings of this study are included within the article.

Conflicts of Interest: The authors declare that they have no competing interest.

\section{References}

1. Hardy, G.H.; Littlewood, J.E.; Polya, G. Inequalities; Cambridge University Press: Cambridge, UK, 1934.

2. Krnić, M.; Pečarić, J. Extension of Hilbert's inequality. J. Math. Anal. Appl. 2006, 324, 150-160. [CrossRef]

3. Yang, B. On a generalization of Hilbert's double series theorem. Math. Inequalities Appl. 2002, 18, 197-204. [CrossRef]

4. Adiyasuren, V.; Batbold, T.; Azar, L.E. A new discrete Hilbert-type inequality involving partial sums. J. Inequalities Appl. 2019, 2019, 127. [CrossRef]

5. Yang, B.C. The Norm of Operator and Hilbert-Type Inequalities; Science Press: Beijing, China, 2009.

6. Krnić, M.; Pečarić, J. General Hilbert's and Hardy's inequalities. Math. Inequal. Appl. 2005, 8, 29-51. [CrossRef]

7. Perić, I.; Vuković, P. Multiple Hilbert's type inequalities with a homogeneous kernel. Banach J. Math. Anal. 2011, 5, 33-43. [CrossRef]

8. Adiyasuren, V.; Batbold, T.; Krnić, M. Hilbert-type inequalities involving differential operators, the best constants, and applications . Math. Inequalities Appl. 2015, 111-124. [CrossRef]

9. Hong, Y.; Wen, Y.M. A necessary and sufficient condition of that Hilbert type series inequality with homogeneous kernel has the best constant factor. Ann. Math. 2016, 37, 329-336.

10. Rassias, M.T.; Yang, B. On half-discrete Hilbert's inequality. Appl. Math. Comput. 2013, 220, 75-93. [CrossRef]

11. Yang, B.C.; Krnić, M. A half-discrete Hilbert-type inequality with a general homogeneous kernel of degree 0. J. Math. Inequal. 2012, 6, 401-417.

12. Rassias, M.T.; Yang, B. A multidimensional half-discrete Hilbert-type inequality and the Riemann zeta function. Appl. Math. Comput. 2013, 225, 263-277. [CrossRef]

13. Rassias, M.T.; Yang, B. On a multidimensional half-discrete Hilbert-type inequality related to the hyperbolic cotangent function. Appl. Math. Comput. 2014, 242, 800-813. [CrossRef]

14. Yang, B.C.; Debnath, L. Half-Discrete Hilbert-Type Inequalities; World Scientific Publishing: Singapore, 2014.

15. Huang, Z.; Yang, B. Equivalent property of a half-discrete Hilbert's inequality with parameters. J. Inequalities Appl. 2018, 2018. [CrossRef] [PubMed]

16. Yang, B.; Wu, S.; Wang, A. On a Reverse Half-Discrete Hardy-Hilbert's Inequality with Parameters. Mathematics 2019, 7, 1054. [CrossRef]

17. Wang, A.-Z.; Yang, B.-C.; Chen, Q. Equivalent properties of a reverse half-discrete Hilbert's inequality. J. Inequalities Appl. 2019, 2019. [CrossRef]

18. Kuang, J.C. Applied Inequalities; Shangdong Science and Technology Press: Jinan, China, 2004. 